\title{
Duration of Suppression of Adrenal Steroids after Glucocorticoid Administration
}

\author{
John S. Fuqua, ${ }^{1}$ Deborah Rotenstein, ${ }^{2}$ and Peter A. Lee ${ }^{1,3}$ \\ ${ }^{1}$ Section of Pediatric Endocrinology and Diabetology, James Whitcomb Riley Hospital for Children, \\ School of Medicine, Indiana University, Indianapolis, IN 46202, USA \\ ${ }^{2}$ Pediatric Alliance, Pittsburgh, PA 15218, USA \\ ${ }^{3}$ The Milton S. Hershey Medical Center, College of Medicine, Pennsylvania State University, \\ Hershey, PA 17033, USA
}

Correspondence should be addressed to John S. Fuqua, jsfuqua@iupui.edu

Received 22 December 2009; Accepted 27 January 2010

Academic Editor: Todd Nebesio

Copyright (๑) 2010 John S. Fuqua et al. This is an open access article distributed under the Creative Commons Attribution License, which permits unrestricted use, distribution, and reproduction in any medium, provided the original work is properly cited.

\begin{abstract}
Hydrocortisone has long been the treatment of choice for congenital adrenal hyperplasia (CAH). However, treatment with this medication remains problematic. Patients with 21-hydroxylase deficiency CAH have significant diurnal variation in the secretion of 17-hydroxyprogesterone (17OHP). When considering treatment strategies, this variation must be considered along with the pharmacokinetic and pharmacodynamic properties of exogenous glucocorticoids. Orally administered hydrocortisone is highly bioavailable, but it has a short time to maximum concentration $\left(\mathrm{T}_{\max }\right)$ and half life $\left(\mathrm{T}_{1 / 2}\right)$. While prednisone has a somewhat longer $\mathrm{T}_{\max }$ and $\mathrm{T}_{1 / 2}$, they remain relatively short. There have been several studies of the pharmacodynamics of hydrocortisone. We present data indicating that the maximum effect of hydrocortisone in $\mathrm{CAH}$ patients is seen 3 hours after a morning dose. After an evening dose, suppression of adrenal hormones continues until approximately 0500 the next day. In both situations, however, there is a large degree of intersubject variability. These data are consistent with earlier published studies. Use of alternate specimen types, possibly in conjunction with delayed release hydrocortisone preparations under development, may allow the practitioner to design a medication regimen that provides improved control of androgen secretion. Whatever dosing strategy is used, clinical judgment is required to ensure the best outcome.
\end{abstract}

\section{Introduction}

Glucocorticoids and mineralocorticoids are mainstays of the treatment of individuals with congenital adrenal hyperplasia $(\mathrm{CAH})$. Several different glucocorticoids are in common use, including hydrocortisone, prednisone/prednisolone, and dexamethasone. Each of these agents has different pharmacokinetic and pharmacodynamic properties, and tailoring their dosing to provide individualized and appropriate control of adrenal androgen secretion remains a major clinical challenge. In this article, we will review the secretory patterns of adrenal steroids and discuss the pharmacokinetics of hydrocortisone and prednisolone. We will then present data from ourselves and others regarding the pharmacodynamics of hydrocortisone and review principles of treatment relating to steroid dosing.

\section{Variability of Cortisol and 17-Hydroxyprogesterone (170HP) Secretion}

When assessing the efficacy of CAH treatment with different doses of glucocorticoids, one must take into account normal diurnal secretory patterns. It has long been known that cortisol production rates range between $5-9 \mathrm{mg} / \mathrm{m}^{2} /$ day. This holds true for neonates, children, adolescents, and adults $[1,2]$. The serum concentration of cortisol in normal individuals with typical sleep-wake patterns begins to rise at about 0400 and reaches its peak between 0700 and 0900 [3]. Over time, this diurnal variation is constant within individuals, although person-to-person variability is large [4]. Similarly, the variation of $17 \mathrm{OHP}$ secretion over a 24-hour period has long been recognized [5, 6]. Untreated subjects with $\mathrm{CAH}$ experience increasing serum 
concentrations of 17OHP between 2400 and 0400. After 1200, 17OHP levels spontaneously fall, although even at their nadir they remain much higher than normal $[7,8]$. In treated patients with $\mathrm{CAH}$, a similar pattern is observed. Charmandari et al. studied steroid secretory patterns in 36 treated children and adolescents with $\mathrm{CAH}$ [9]. They divided the 24-hour day into two periods: daytime, defined as 04001600, and nighttime, defined as 1600-0400. Mean 17OHP levels for 19 individuals who were relatively unsuppressed were $5296 \mathrm{ng} / \mathrm{dL}$ during the daytime and $2350 \mathrm{ng} / \mathrm{dL}$ during the nighttime. In the 17 subjects who were more completely suppressed, this diurnal variation was present but not as apparent. In this group, daytime 17OHP concentrations averaged $59 \mathrm{ng} / \mathrm{dL}$, and nighttime concentrations averaged $36 \mathrm{ng} / \mathrm{dL}$.

\section{Pharmacokinetics of Commonly Used Glucocorticoids}

An understanding of the pharmacokinetics of hydrocortisone and prednisone/prednisolone is helpful when planning a treatment regimen for $\mathrm{CAH}$, particularly in regards to the timing of multiple doses and when adjusting doses to improve control.

3.1. Hydrocortisone. Hydrocortisone is rapidly absorbed after an oral dose, and concomitant food intake does not appear to prevent absorption [10]. Hydrocortisone has a high degree of intestinal permeability, crossing the intestinal mucosa in a passive fashion. The bioavailability after oral administration is high, $>90 \%$ [11], and the time to peak concentration $\left(\mathrm{T}_{\max }\right)$ is roughly $1-2$ hours. However, this depends on the exact formulation administered, because it is thought that the rate dependent step in absorption is the dissolution of hydrocortisone tablets [10]. The elimination half-life $\left(\mathrm{T}_{1 / 2}\right)$ of hydrocortisone is also short, 1.8-2 hours following either oral or intravenous administration. The pharmacokinetics of hydrocortisone are nonlinear, in part due to extensive protein binding. As a result, higher doses result in more rapid clearance rates [11]. Hence, doubling the dose does not lead to doubling of the area under the curve of plasma hydrocortisone versus time. A typical pharmacokinetic profile of hydrocortisone is shown in Figure 1.

3.2. Prednisone/Prednisolone. Similar to hydrocortisone, prednisone is also rapidly and nearly completely absorbed after an oral dose. However, prednisone itself is biologically inactive, and before having any effect, its 11-keto group must be reduced by hepatic 11- $\beta$-hydroxysteroid dehydrogenase to form the active drug, prednisolone. Although this transformation occurs rapidly, it does account for the delayed $\mathrm{T}_{\max }$ of prednisone versus prednisolone (2.6 hours versus 1.3 hours, resp.) [11]. There is bidirectional interconversion of prednisone and prednisolone, with $76 \%$ recycling, although the enzyme kinetics favor the formation of prednisolone [13]. The $\mathrm{T}_{1 / 2}$ of both prednisone and prednisolone have been reported to be 3-4 hours. Studies

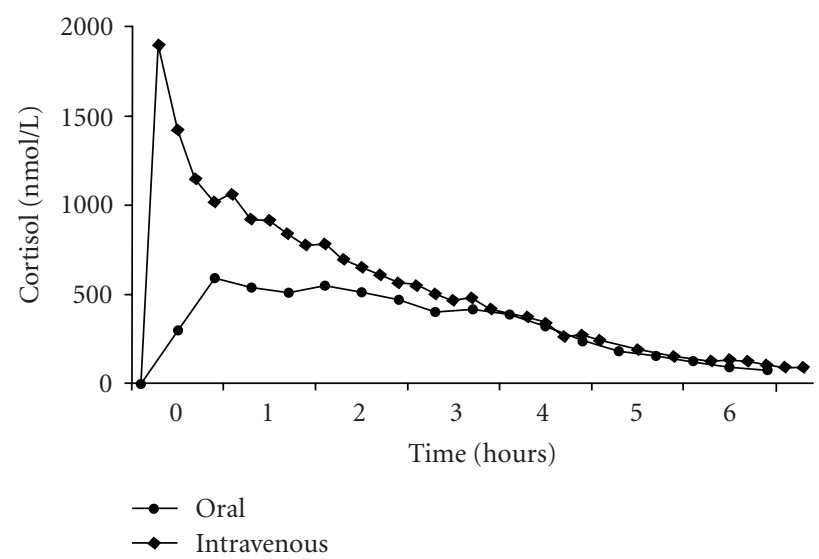

FIGURE 1: Pharmacokinetic profile following the morning administration of intravenous and oral doses of hydrocortisone in 16 subjects with $\mathrm{CAH}$, at a median age of 10.6 years. Figure taken from [12]. Society for Endocrinology, 2001. Used with permission.

in children suggest that the $\mathrm{T}_{1 / 2}$ may be shorter, 2.2 hours $[11,14]$, possibly due to a more rapid clearance rate [15]. The kinetics do not appear to be altered by gastrointestinal disease or renal transplant status [16, 17], but untreated hypothyroidism leads to a dramatic decrease in clearance [18]. Prednisone/prednisolone has complex, nonlinear pharmacokinetics due to protein binding which becomes saturated at higher doses. This leads to increasing clearance rates and a decrease in the peak concentration achieved per milligram dose. However, this phenomenon has been recognized at doses well above those used for nonstressed CAH patients [11].

\section{Pharmacodynamics of Commonly Used Glucocorticoids}

When used to treat $\mathrm{CAH}$, both the pharmacokinetics and pharmacodynamics of steroid preparations must be considered. Superimposed on the pharmacokinetics are the diurnal variations in ACTH, cortisol, 17OHP, androstenedione, and testosterone secretion. Thus, timing of administration of the steroid relative to adrenal hormone secretion becomes a critical factor when interpreting subsequent levels. There have been limited studies of the effectiveness of hydrocortisone administration at suppressing endogenous hormone concentrations in subjects with $\mathrm{CAH}[8,9,19,20]$. The studies have been performed almost exclusively in individuals receiving hydrocortisone treatment, with only a few subjects receiving prednisone.

We studied 16 subjects (9 males) with 21-hydroxylase deficiency $\mathrm{CAH}$ at a mean age of $12.2 \pm 5$ years who were receiving hydrocortisone three times daily [19]. Institutional review board approval and informed consent/assent were obtained. After an overnight admission with their usual evening hydrocortisone dose, subjects were administered their usual morning dose of steroid after obtaining baseline serum concentrations of 17OHP, androstenedione, and 


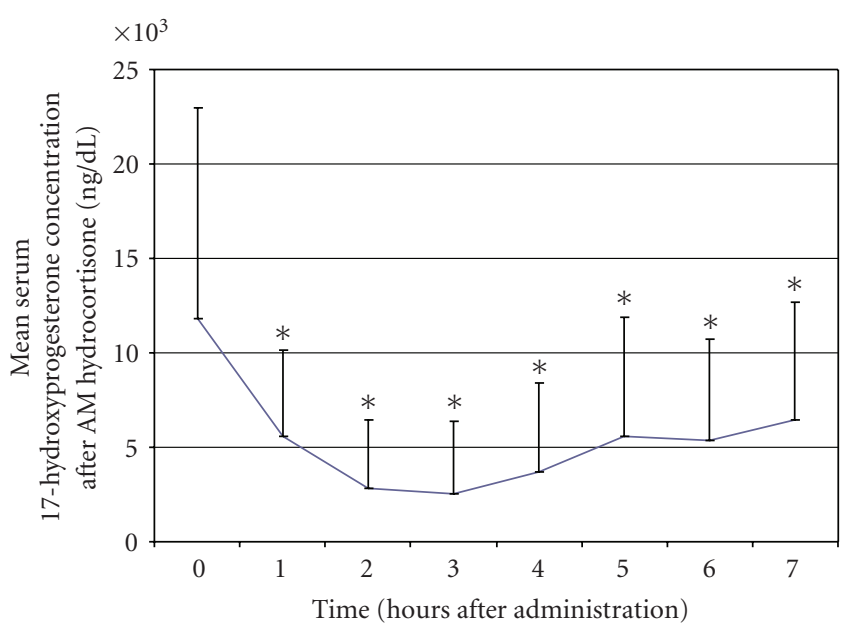

(a)

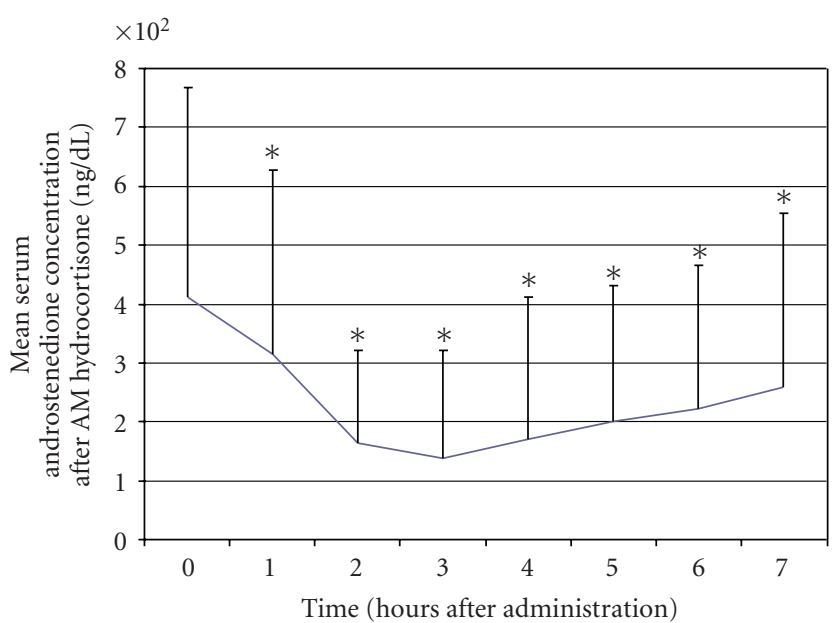

(b)

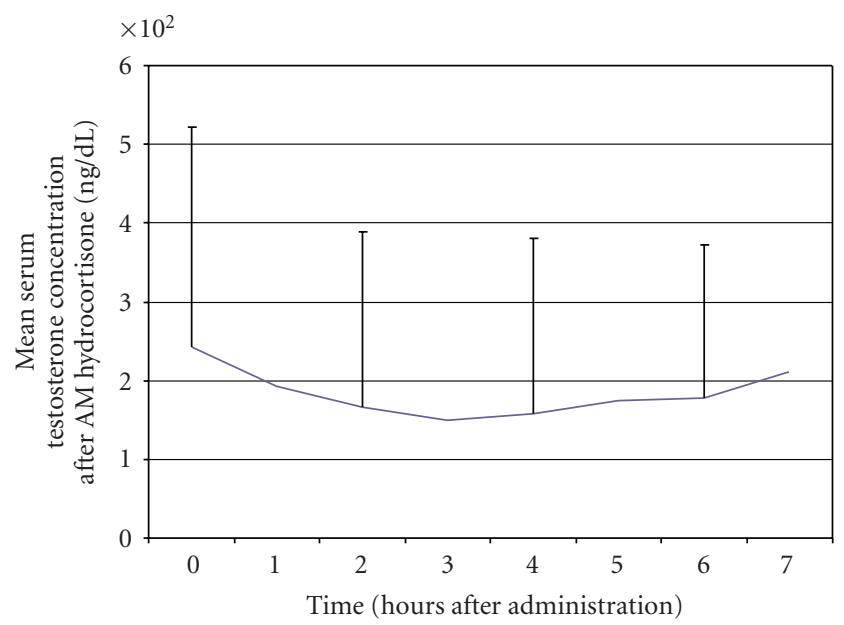

(c)

FIGURE 2: Serum concentrations of 17-hydroxyprogesterone, androstenedione, and testosterone after administration of hydrocortisone in the morning. ${ }^{*} P<.05$ compared to baseline.

testosterone. Following steroid administration at times ranging from 0700 to 0830 , serial measurements of serum 17OHP and androstenedione (hourly) and testosterone (every two hours) were made. Baseline concentrations of $17 \mathrm{OHP}$ and androstenedione were quite elevated at $12,657 \mathrm{ng} / \mathrm{dL}$ and $311 \mathrm{ng} / \mathrm{dL}$, respectively. Maximum suppression of $17 \mathrm{OHP}$ and androstenedione occurred 3 hours after administration of steroid, and levels of both substances then began to return gradually towards baseline (Figure 2). Seven hours after administration of steroid, 17OHP concentrations remained at $59 \%$ of baseline, while androstenedione concentrations were at $62 \%$ of baseline. Serum testosterone concentrations were also elevated at baseline and then appeared to decline slightly, with a nadir three hours after steroid dosing, but this did not reach statistical significance.

A similar study was conducted on a different group of six subjects to assess further the overnight suppression of adrenal function. Subjects were given their usual evening doses of hydrocortisone and then had serum concentrations of androstenedione, 17OHP, and testosterone measured every two hours from 2100 until 0900 the next morning. (Figure 3) In the six subjects in whom androstenedione was measured, concentrations were stable and relatively low until 0300. By 0500, androstenedione concentrations had begun to increase steadily until the end of the study at 0900 . Five of the subjects had 17OHP and testosterone measured according to the same protocol. 17OHP concentrations at baseline averaged $665 \mathrm{ng} / \mathrm{dL}$ and declined to $324 \mathrm{ng} / \mathrm{dL}$ after two hours. Levels stayed suppressed until 0500, when they increased to $2625 \mathrm{ng} / \mathrm{dL}$, and then continued to increase steadily until 0900, at which time the study stopped. Testosterone concentrations, by contrast, did not show a significant decline after administration of hydrocortisone, and they remained stable through the night and into the morning. Although there are clear trends in the patterns of overnight secretion, most of the data were not statistically different because of the small number of subjects.

During both the daytime and overnight studies, there was a large degree of variability among subjects for all three hormones, both at baseline and during the monitoring 


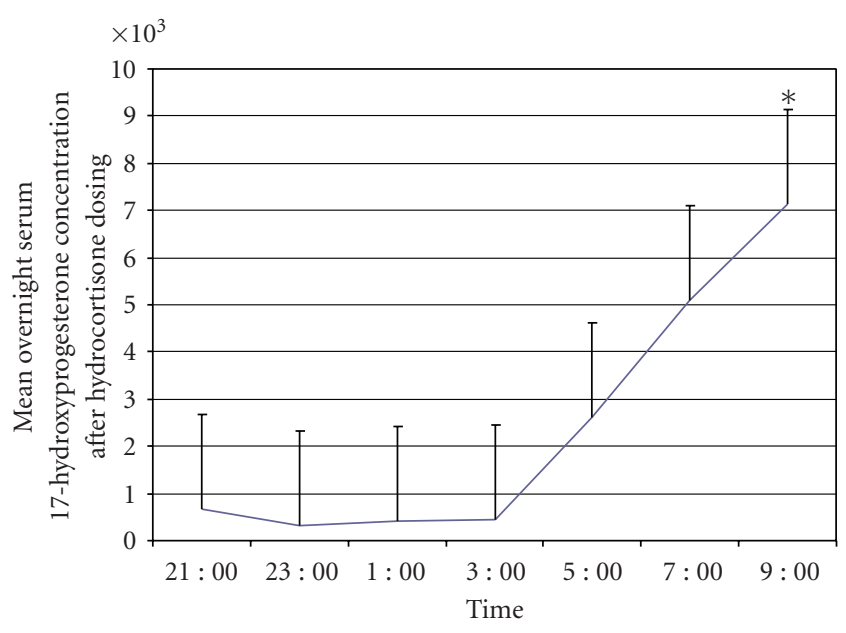

(a)

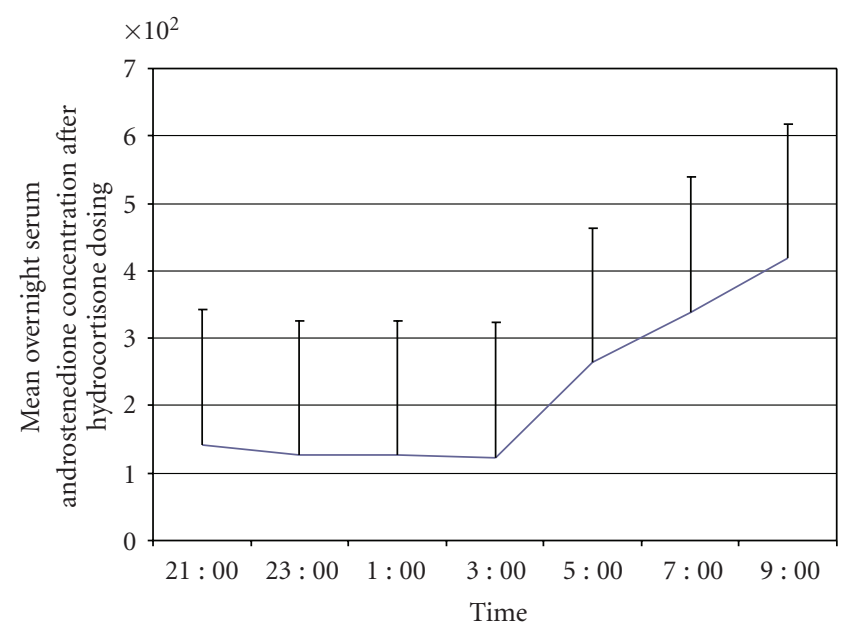

(b)

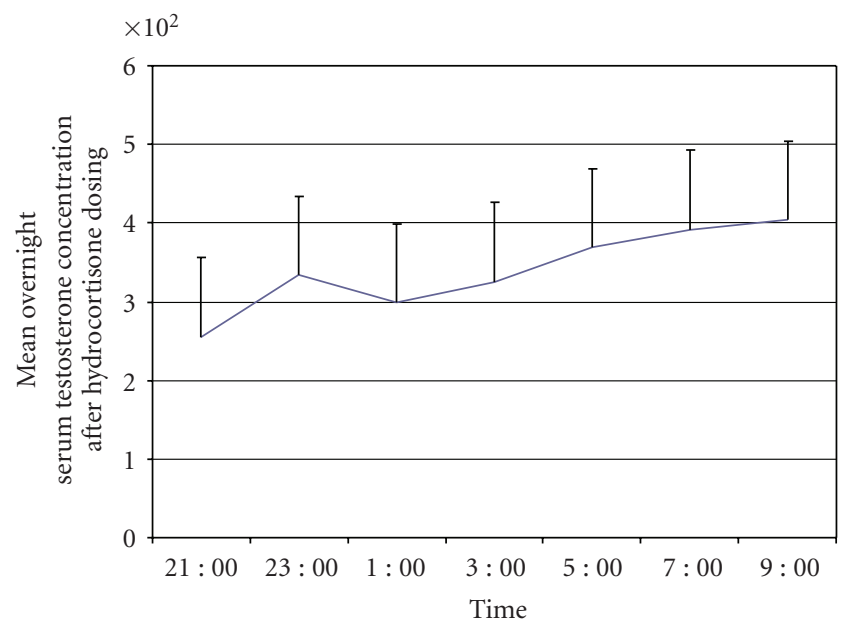

(c)

FIGURE 3: Serum concentrations of 17-hydroxyprogesterone, androstenedione, and testosterone after administration of a nighttime dose of hydrocortisone. ${ }^{*} P=.05$ compared to baseline.

periods. This is similar to the variability noted in other studies $[8,9,20]$.

Thus, after administration of steroid to subjects with $\mathrm{CAH}$ at night, when hypothalamic-pituitary-adrenal (HPA) axis activity is relatively low, subjects maintained $17 \mathrm{OHP}$ and androstenedione levels in a low, stable range for approximately eight hours, until 0500. In contrast, administration of steroid in the morning, during peak HPA axis activity, resulted in a short-lived suppression of adrenal activity lasting only three hours. Thus, the timing of measurement of 17OHP relative to the 24-hour clock and the timing relative to dosing of glucocorticoid are both critical for the interpretation of pharmacodynamic data.

There have been several previous reports of the pharmacodynamics of hydrocortisone use in patients with $\mathrm{CAH}$. In an older study, Frisch et al. [8] studied 11 prepubertal and pubertal children, seven of whom were receiving hydrocortisone. Subjects had a rapid suppression of $17 \mathrm{OHP}$ after the morning dose and a mid-day dose 5 hours later. They received an evening dose at 1830 , and $17 \mathrm{OHP}$ concentrations began to rise between 2400 and 0200 . Testosterone concentrations remained suppressed during the day, but increased in most subjects after 0200. Thus, their results differed from ours in that the rise in HPA axis activity seemed to occur earlier and testosterone levels showed a diurnal variation.

Charmandari et al. [9] studied 36 prepubertal and pubertal subjects with $\mathrm{CAH}$, all of whom were receiving hydrocortisone. They obtained samples at 20 minute intervals over a 24 -hour time period. Morning administration of hydrocortisone also resulted in a rapid drop of $17 \mathrm{OHP}$ concentrations, with maximum suppression occurring one hour after peak cortisol levels were attained. The majority of their subjects received hydrocortisone twice daily, with only 39\% receiving it in three divided doses. Mean serum $17 \mathrm{OHP}$ concentrations during the day were stable in the total group and declined further after the evening dose of hydrocortisone at 2100-2200. In their subjects, $17 \mathrm{OHP}$ began to rise again at 0400 . Hence, their data are consistent 
with ours regarding the time course of suppression in the morning and escape overnight. They did not compare the twice-daily hydrocortisone group to the three-times-daily group.

In a study of the salivary secretion of cortisol and 17OHP, Gröschl et al. [20] evaluated 10 normal adult controls and 10 children with $\mathrm{CAH}$ and found that hydrocortisone administered at 0700 led to a rapid reduction in salivary $17 \mathrm{OHP}$ levels, reaching a nadir at three hours. Thereafter, levels of 17OHP began to increase steadily, although they remained below baseline at five hours. They also noted significant variability in response to hydrocortisone among subjects. Again, these data are similar to ours. These authors also noted a more rapid clearance of hydrocortisone from salivary samples in affected subjects $\left(\mathrm{T}_{1 / 2}=56.4\right.$ minutes) compared to normal controls $\left(\mathrm{T}_{1 / 2}=94.5\right.$ minutes $)$.

There have been limited studies of the pharmacodynamics of prednisone use in individuals with CAH. Huseman et al. evaluated the ability of prednisone to control $\mathrm{CAH}$ when administered at different frequencies. It appeared that when a single daily dose of prednisone was administered at 2000 hours, the 17OHP concentration had escaped from suppression by 0900 the next morning in four of their six subjects. Unfortunately, they did not measure $17 \mathrm{OHP}$ levels at any other times, so the duration of action could not be evaluated further [21]. In a study by Zipf et al., a group of six subjects who were being treated with prednisone had hourly measurements of serum 17OHP concentrations [22]. Five of the six subject did not demonstrate escape from suppression. All of the subjects were either quite short or had short adult heights, suggesting that they were being chronically over treated. The subject who did escape suppression appeared to do so at about 0500 , following a midnight dose of prednisone. Four subjects reported by Frisch et al. [8], appeared to escape suppression between 2400 and 0500 after an 1800 dose of prednisone given the day before. We studied four subjects receiving prednisone who were not included in the above analysis, and based on these very limited data, there was no difference from those receiving hydrocortisone, although any differences may be difficult to identify given the high subject-to-subject variability.

\section{Methods Used to Assess Control of CAH}

The degree of suppression of adrenal metabolites is most often assessed by measuring the concentrations of 17OHP, androstenedione, and/or testosterone. These substances are most commonly measured in serum, but 17OHP has also been measured in filter paper blood spots and in saliva. Historically, studies have used radioimmunoassays (RIAs), but high performance liquid chromatography-tandem mass spectrometry techniques (HPLC-MSMS) have more recently been applied to the measurement of these analytes. HPLCMSMS offers the advantage of significant increases in the specificity of the assay, which may be relatively low in some RIAs, particularly if performed in the newborn period. Because of the increased assay specificity, concentrations of these substances may be reported as lower when measured by HPLC-MSMS than by RIA. This makes it difficult to compare recent data using modern assay techniques to older data, especially those using less specific polyclonal RIAs.

Data from filter paper blood spots show good correlation with results obtained simultaneously from serum, with serum levels generally corresponding to those from filter paper spots. Additionally, there appears to be little degradation related to storage time or temperature over a two-week period $[23,24]$.

$17 \mathrm{OHP}$ is present in saliva in concentrations far lower than in serum. Older techniques to measure salivary $17 \mathrm{OHP}$ used RIAs that were initially developed for use with serum and subsequently modified to use with saliva [20,25]. Newer techniques have taken advantage of the higher specificity of HPLC-MSMS to measure 17OHP in saliva [26]. Correlation between serum and salivary 17OHP using modified RIAs is variable $(r=0.62-0.93),[25,27]$ while the correlation using HPLC-MSMS appears to be higher $(r=0.98)$, [26] likely related to increased specificity. Additionally, 17OHP concentrations in saliva were $3-4 \%$ of those in serum using the modified RIAs, but only $0.5 \%$ when the HPLC-MSMS technique was employed. This again probably reflects the increased specificity of mass spectrometry.

\section{Optimizing the Dosing Schedule for CAH}

Despite the availability of longer acting glucocorticoids such as prednisone and dexamethasone, hydrocortisone remains the drug of choice for treatment of children with $\mathrm{CAH}$ according to the position statement by the Lawson Wilkins Pediatric Endocrine Society and the European Society for Pediatric Endocrinology [28] and the forthcoming clinical practice guideline from The Endocrine Society. This is because of the higher potential for growth suppression with longer acting, more potent steroids [28, 29]. Recommendations for dosing vary, but current data suggest that the daily dose of hydrocortisone during puberty should be kept below $17 \mathrm{mg} / \mathrm{m}^{2}$ if possible, in order to minimize its growth suppressive effects [30]. Unfortunately, the relatively short half life of hydrocortisone leads to difficulties suppressing adrenal androgen production, particularly early in the morning as seen in the data presented above [31]. This has led to different opinions regarding how best to approach the administration of hydrocortisone. As discussed above, 17OHP and androstenedione reach their highest concentrations in serum early in the morning, and some authors have recommended administering hydrocortisone in split doses with the largest share given early in the morning [9]. While this will lead to rapid suppression of the high $17 \mathrm{OHP}$ and androstenedione levels, it does not address the increasing concentrations of adrenal hormones that begins at about 0400 . Others have recommended giving a larger dose of hydrocortisone at bedtime, with the expectation that this dose will suppress, at least partially, the early AM surge $[32,33]$. The effectiveness of this is uncertain given the short $\mathrm{T}_{1 / 2}$ of hydrocortisone and the need for relatively high concentrations of cortisol to suppress endogenous steroid production in the early morning. Additionally, circulating levels of cortisol overnight are normally quite low, and in patients with $\mathrm{CAH}$ much less hydrocortisone is required 
to suppress adrenal steroid production in the timeframe of 2200-0200. Giving a large hydrocortisone dose at bedtime thus potentially exposes the patient to unnecessarily high levels during this window. At the present time, there is no consensus on this point, and practice guidelines and position statements do not recommend specific dosing strategies to address this.

In attempts to tailor hydrocortisone administration to the specific needs of patients with $\mathrm{CAH}$, sustained release preparations have been developed. Two recent publications have presented data on these preparations. Johannsson et al. [10] studied a dual release hydrocortisone tablet that had a rapidly released coating surrounding a sustained release core. Using this preparation once daily in the morning, they were able to duplicate peak cortisol concentrations with gradually declining levels during the day and very low concentrations overnight. However, this preparation did not duplicate the early morning increase in cortisol between 0400 and the time of medication administration. While this profile may be suited to patients with adrenal insufficiency, it may not be optimal for those with $\mathrm{CAH}$, as adrenal metabolite production would already be near its peak when the dose is given. Debono et al. [34] studied a different sustained release hydrocortisone preparation which had a time to peak concentration in healthy subjects of 7-8 hours, compared to 1.8 hours for standard hydrocortisone tablets. This allowed for a three-hour lag after administration before the serum concentration of cortisol began to increase. Using these data for varying doses of the sustained release preparation, they simulated administering a dose at 2300 and another dose at 0700 . This resulted in a pattern that mimicked the normal diurnal variation of cortisol concentration, with serum cortisol levels being low from 2300 to 0300 , increasing to reach a peak level at 0700 , and then gradually declining back to baseline. This medication has not been studied in subjects with CAH, but it offers promise for a tailored therapy that potentially could improve control and reduce the incidence of long-term growth complications of the condition and its treatment.

There has been limited experience in the delivery of hydrocortisone by continuous subcutaneous infusion using an insulin pump, but this is also has the potential for fitting the administration of hydrocortisone to physiologic requirements for those with $\mathrm{CAH}[35,36]$. One of the authors (DR) has achieved excellent control of adrenal steroid precursors without apparent deleterious effects on growth by administering a small dose of prednisone in the evening to provide longer-term overnight and early morning suppression and then giving hydrocortisone during the day to suppress daytime adrenal hormones.

It has long been known that there is a spectrum of salt loss in those with $\mathrm{CAH}$, including those who clinically have the simple virilizing form. These individuals may have elevated levels of plasma renin activity despite normal electrolyte concentrations, indicating subclinical hypovolemia. Treatment of these patients with mineralocorticoid reduces the hypovolemic drive for ACTH secretion and can lower the amount of glucocorticoid needed to appropriately suppress adrenal function. This was demonstrated in 1977 by Rosler et al., who found that treatment of nonsalt wasting patients normalized ACTH secretion and led to further suppression of adrenal steroid secretion [37]. In a later study, Jansen et al. demonstrated that institution of mineralocorticoid therapy in five subjects with $\mathrm{CAH}$ allowed for a decrease in the mean dose of hydrocortisone from $26 \mathrm{mg} / \mathrm{m}^{2} /$ day to $17.6 \mathrm{mg} / \mathrm{m}^{2} /$ day and resulted in improved linear growth [38]. In a retrospective study of the influences of CAH treatment on adult height, patients treated with mineralocorticoid were taller than untreated patients with similar disease severities [39]. Thus, consideration should be given to treating both the salt wasting and simple virilizing forms of CAH with $9 \alpha$ fludrocortisone.

\section{Measuring Control of CAH}

Similar to the different dosing strategies for hydrocortisone, there are different strategies for monitoring the adequacy of control. First and foremost, clinical judgment must be used. This encompasses assessments of height velocity, status of pubertal development, weight gain, skeletal maturation, and adherence to the medication regimen. Any biochemical assessments must be considered in their clinical context. Typically, serum concentrations of 17-hydroxyprogestereone, androstenedione, and testosterone are used as an aid to judge the adequacy of biochemical control. In prepubertal children and in adolescent and adult females, androstenedione and testosterone concentrations are thought to correlate better with adrenal suppression than 17OHP levels. In pubertal males, testosterone levels are not helpful. [40] Laboratory studies may be obtained as a first morning sample or as a random sample at the time of the patient's follow-up appointment. Although the latter strategy offers convenience for the patient and physician, the sample may be obtained at different times relative to the last steroid dose and at different times of the day, resulting in inconsistent assessments of the degree of suppression. For this reason, it is often recommended that biochemical measurements be done in the morning, with consistent timing relative to the last steroid dose $[28,32,41]$. In either case, the time of administration of the previous steroid dose should be noted and taken into consideration when interpreting the results. Additional approaches mentioned above include at-home collection of filter paper blood spots or saliva. These strategies offer the advantage of being able to collect multiple specimens at different times of day, both before and after steroid dosing $[23,24]$. They potentially allow for individual titration of each dose of steroid, again with the goal of giving the amount needed to adequately suppress adrenal function, but not to overly suppress it. A strategy such as this would also enable the physician to adjust the steroid dose to allow for the person to person variation in glucocorticoid clearance rates and individual pharmacodynamics. Unfortunately, assays of 17hydroxyprogesterone and androstenedione in saliva are not widely available in the U.S. Although newborn screening for CAH using filter paper blood spots is performed universally in the U.S., this technology is also not currently commercially available for routine follow-up studies of patients already diagnosed with $\mathrm{CAH}$. 


\section{Conclusions}

In summary, hydrocortisone is the glucocorticoid usually recommended for the treatment of children with CAH. Its rapid onset of action makes it useful for the rapid suppression of adrenal androgen secretion, but its relatively short half life allows for escape from suppression, particularly in the early morning after nighttime administration. Data show that 17OHP and androstenedione concentrations begin to rise at $0300-0500$ when hydrocortisone is given at 2100-2200. Morning administration of hydrocortisone results in rapid suppression of $17 \mathrm{OHP}$ and androstenedione, but because of high morning HPA axis activity, escape occurs after 3-4 hours, requiring a mid-day dose to maintain suppression. Measurement of serum $17 \mathrm{OHP}$ and androstenedione concentrations 3-4 hours after the morning dose of hydrocortisone will demonstrate maximal adrenal suppression. Measurement of adrenal hormones in saliva or in dried filter paper blood spots would allow a more complete profile of $\mathrm{CAH}$ control, and we encourage the commercial development of these approaches. Although prednisone/prednisolone has a longer half life, its pharmacodynamics have not been well studied in children with CAH.

Hydrocortisone dosing recommendations include administering the largest dose in the evening or in the morning, but neither one is likely to provide optimum control. Giving the evening dose of hydrocortisone as late as possible and the morning dose as early as possible, with a mid-day dose 4-5 hours after the morning dose may offer the best compromise. Sustained or delayed release hydrocortisone preparations are in development and offer promise for CAH patients. The combination of different glucocorticoid preparations with different pharmacologic profiles merits further study, analogous to the approach used to treat diabetics with multiple insulin preparations.

\section{References}

[1] D. L. Metzger, N. M. Wright, J. D. Veldhuis, A. D. Rogol, and J. R. Kerrigan, "Characterization of pulsatile secretion and clearance of plasma cortisol in premature and term neonates using deconvolution analysis," Journal of Clinical Endocrinology and Metabolism, vol. 77, no. 2, pp. 458-463, 1993.

[2] J. R. Kerrigan, J. D. Veldhuis, S. A. Leyo, A. Iranmanesh, and A. D. Rogol, "Estimation of daily cortisol production and clearance rates in normal pubertal males by deconvolution analysis," Journal of Clinical Endocrinology and Metabolism, vol. 76, no. 6, pp. 1505-1510, 1993.

[3] K. H. Darzy and S. M. Shalet, "Absence of adrenocorticotropin (ACTH) neurosecretory dysfunction but increased cortisol concentrations and production rates in ACTH-replete adult cancer survivors after cranial irradiation for nonpituitary brain tumors," Journal of Clinical Endocrinology and Metabolism, vol. 90, no. 9, pp. 5217-5225, 2005.

[4] B. Selmaoui and Y. Touitou, "Reproducibility of the circadian rhythms of serum cortisol and melatonin in healthy subjects: a study of three different 24 -h cycles over six weeks," Life Sciences, vol. 73, no. 26, pp. 3339-3349, 2003.

[5] S. M. Atherden, N. D. Barnes, and D. B. Grant, "Circadian variation in plasma 17-hydroxyprogesterone in patients with congenital adrenal hyperplasia," Archives of Disease in Childhood, vol. 47, no. 254, pp. 602-604, 1972.

[6] J. P. Gutai, W. J. Meyer III, A. A. Kowarski, and C. J. Migeon, "Twenty four hour integrated concentrations of progesterone, 17 hydroxyprogesterone and cortisol in normal male subjects," Journal of Clinical Endocrinology and Metabolism, vol. 44, no. 1, pp. 116-120, 1977.

[7] J. Solyom, "Diurnal variation in blood 17-hydroxyprogesterone concentrations in untreated congenital adrenal hyperplasia," Archives of Disease in Childhood, vol. 59, no. 8, pp. 743747, 1984.

[8] H. Frisch, K. Parth, E. Schober, and W. Swoboda, "Circadian patterns of plasma cortisol, 17-hydroxyprogesterone, and testosterone in congenital adrenal hyperplasia," Archives of Disease in Childhood, vol. 56, no. 3, pp. 208-213, 1981.

[9] E. Charmandari, D. R. Matthews, A. Johnston, C. G. D. Brook, and P. C. Hindmarsh, "Serum cortisol and 17hydroxyprogesterone interrelation in classic 21-hydroxylase deficiency: is current replacement therapy satisfactory?" Journal of Clinical Endocrinology and Metabolism, vol. 86, no. 10, pp. 4679-4685, 2001.

[10] G. Johannsson, R. Bergthorsdottir, A. G. Nilsson, H. Lennernas, T. Hedner, and S. Skrtic, "Improving glucocorticoid replacement therapy using a novel modified-release hydrocortisone tablet: a pharmacokinetic study," European Journal of Endocrinology, vol. 161, no. 1, pp. 119-130, 2009.

[11] D. Czock, F. Keller, F. M. Rasche, and U. Haussler, "Pharmacokinetics and pharmacodynamics of systemically administered glucocorticoids," Clinical Pharmacokinetics, vol. 44, no. 1, pp. 61-98, 2005.

[12] E. Charmandari, A. Johnston, C. G. D. Brook, and P. C. Hindmarsh, "Bioavailability of oral hydrocortisone in patients with congenital adrenal hyperplasia due to 21-hydroxylase deficiency," Journal of Endocrinology, vol. 169, no. 1, pp. 6570, 2001.

[13] V. Garg and W. J. Jusko, "Bioavailability and reversible metabolism of prednisone and prednisolone in man," Biopharmaceutics and Drug Disposition, vol. 15, no. 2, pp. 163-172, 1994.

[14] O. C. Green, R. J. Winter, F. S. Kawahara, et al., "Plasma levels, half-life values, and correlation with physiologic assays for growth and immunity," Journal of Pediatrics, vol. 93, no. 2, pp. 299-303, 1978.

[15] M. R. Hill, S. J. Szefler, B. D. Ball, M. Bartoszek, and A. M. Brenner, "Monitoring glucocorticoid therapy: a pharmacokinetic approach," Clinical Pharmacology and Therapeutics, vol. 48, no. 4, pp. 390-398, 1990.

[16] C. Chavatte, G. Guest, V. Proust, et al., "Glucocorticoid pharmacokinetics and growth retardation in children with renal transplants," Pediatric Nephrology, vol. 19, no. 8, pp. 898904, 2004.

[17] C. Faure, J. André, C. Pelatan, et al., "Pharmacokinetics of intravenous methylprednisolone and oral prednisone in paediatric patients with inflammatory bowel disease during the acute phase and in remission," European Journal of Clinical Pharmacology, vol. 54, no. 7, pp. 555-560, 1998.

[18] S. Bergamaschi, R. Rusconi, M. Gervasoni, A. E. Rigamonti, S. Cella, and S. R. Bareggi, "Pharmacokinetics of prednisone and prednisolone in a case of hypothyroidism: effect of replacement therapy," Steroids, vol. 70, no. 11, pp. 787-789, 2005.

[19] D. Rotenstein, W. Hecker, and P. A. Lee, "Adrenal steroid levels after oral glucocorticoid in congenital adrenal hyperplasia," Pediatric Research, vol. 17, p. 171A, 1983. 
[20] M. Gröschl, M. Rauh, and H. G. Dörr, "Cortisol and 17hydroxyprogesterone kinetics in saliva after oral administration of hydrocortisone in children and young adolescents with congenital adrenal hyperplasia due to 21-hydroxylase deficiency," Journal of Clinical Endocrinology and Metabolism, vol. 87, no. 3, pp. 1200-1204, 2002.

[21] C. A. Huseman, M. M. Varma, R. M. Blizzard, and A. Johanson, "Treatment of congenital virilizing adrenal hyperplasia patients with single and multiple daily doses of prednisone," Journal of Pediatrics, vol. 90, no. 4, pp. 538-542, 1977.

[22] W. B. Zipf, G. E. Bacon, and R. P. Kelch, "Hormonal and clinical responses to prednisone treatment in adolescents with congenital adrenal hyperplasia," Hormone Research, vol. 12, no. 4, pp. 206-217, 1980.

[23] H. H. Bode, S. A. Rivkees, D. M. Cowley, K. Pardy, and S. Johnson, "Home monitoring of 17 hydroxyprogesterone levels in congenital adrenal hyperplasia with filter paper blood samples," Journal of Pediatrics, vol. 134, no. 2, pp. 185-189, 1999.

[24] I. Shimon, I. Kaiserman, and J. Sack, "Home monitoring of $17 \alpha$-hydroxyprogesterone levels by filter paper blood spots in patients with 21-hydroxylase deficiency," Hormone Research, vol. 44, no. 6, pp. 247-252, 1995.

[25] P. G. Mylonas, M. Makri, N. A. Georgopoulos, et al., "Adequacy of saliva 17-hydroxyprogesterone determination using various collection methods," Steroids, vol. 71, no. 3, pp. 273-276, 2006.

[26] Y. Shibayama, T. Higashi, K. Shimada, et al., "Liquid chromatography-tandem mass spectrometric method for determination of salivary $17 \alpha$-hydroxyprogesterone: a noninvasive tool for evaluating efficacy of hormone replacement therapy in congenital adrenal hyperplasia," Journal of Chromatography B, vol. 867, no. 1, pp. 49-56, 2008.

[27] O. Arisaka, N. Shimura, Y. Nakayama, M. Arisaka, and K. Yabuta, "Salivary concentration of 17-hydroxyprogesterone in monitoring treatment of congenital adrenal hyperplasia," Deutsche Medizinische Wochenschrift, vol. 113, no. 49, pp. 1913-1915, 1988.

[28] "Consensus statement on 21-hydroxylase deficiency from the Lawson Wilkins Pediatric Endocrine Society and the European Society for Paediatric Endocrinology," Journal of Clinical Endocrinology and Metabolism, vol. 87, no. 9, pp. 4048-4053, 2002.

[29] W. Bonfig, S. Bechtold, H. Schmidt, D. Knorr, and H. P. Schwarz, "Reduced final height outcome in congenital adrenal hyperplasia under prednisone treatment: deceleration of growth velocity during puberty," Journal of Clinical Endocrinology and Metabolism, vol. 92, no. 5, pp. 1635-1639, 2007.

[30] W. Bonfig, S. B. D. Pozza, H. Schmidt, P. Pagel, D. Knorr, and H. P. Schwarz, "Hydrocortisone dosing during puberty in patients with classical congenital adrenal hyperplasia: an evidence-based recommendation," Journal of Clinical Endocrinology and Metabolism, vol. 94, no. 10, pp. 3882-3888, 2009.

[31] M. Debono, R. J. Ross, and J. Newell-Price, "Inadequacies of glucocorticoid replacement and improvements by physiological circadian therapy," European Journal of Endocrinology, vol. 160, no. 5, pp. 719-729, 2009.

[32] D. P. Merke, "Approach to the adult with congenital adrenal hyperplasia due to 21-hydroxylase deficiency," Journal of Clinical Endocrinology and Metabolism, vol. 93, no. 3, pp. 653$660,2008$.
[33] R. L. Rosenfield, "Serum cortisol and 17-hydroxyprogesterone concentrations in children with classic congenital adrenal hyperplasia," Journal of Clinical Endocrinology and Metabolism, vol. 87, no. 6, p. 2993, 2002.

[34] M. Debono, C. Ghobadi, A. Rostami-Hodjegan, et al., "Modified-release hydrocortisone to provide circadian cortisol profiles," Journal of Clinical Endocrinology and Metabolism, vol. 94, no. 5, pp. 1548-1554, 2009.

[35] S. M. Bryan, J. W. Honour, and P. C. Hindmarsh, "Management of altered hydrocortisone pharmacokinetics in a boy with congenital adrenal hyperplasia using a continuous subcutaneous hydrocortisone infusion," Journal of Clinical Endocrinology and Metabolism, vol. 94, no. 9, pp. 3477-3480, 2009.

[36] Z. Merza, A. Rostami-Hodjegan, A. Memmott, et al., "Circadian hydrocortisone infusions in patients with adrenal insufficiency and congenital adrenal hyperplasia," Clinical Endocrinology, vol. 65, no. 1, pp. 45-50, 2006.

[37] A. Rosler, L. S. Levine, B. Schneider, M. Novogroder, and M. I. New, "The interrelationship of sodium balance, plasma renin activity and ACTH in congenital adrenal hyperplasia," Journal of Clinical Endocrinology and Metabolism, vol. 45, no. 3, pp. 500-512, 1977.

[38] M. Jansen, J. M. Wit, and J. L. van den Brande, "Reinstitution of mineralocorticoid therapy in congenital adrenal hyperplasia. Effects on control and growth," Acta Paediatrica Scandinavica, vol. 70, no. 2, pp. 229-233, 1981.

[39] A. Balsamo, A. Cicognani, L. Baldazzi, et al., "CYP21 genotype, adult height, and pubertal development in 55 patients treated for 21-hydroxylase deficiency," Journal of Clinical Endocrinology and Metabolism, vol. 88, no. 12, pp. 5680-5688, 2003.

[40] P. A. Lee, M. D. Urban, J. P. Gutai, and C. J. Migeon, "Plasma progesterone, 17-hydroxyprogesterone, androstenedione and testosterone in prepubertal, pubertal and adult subjects with congenital virilizing adrenal hyperplasia as indicators of adrenal suppression," Hormone Research, vol. 13, no. 6, pp. 347-357, 1980.

[41] P. C. Hindmarsh, "Management of the child with congenital adrenal hyperplasia," Best Practice and Research: Clinical Endocrinology and Metabolism, vol. 23, no. 2, pp. 193-208, 2009. 